\title{
What's the Matter with Distal Deep Vein Thrombosis?
}

\author{
Stefano Barco ${ }^{1}$ (1) \\ ${ }^{1}$ Center for Thrombosis and Hemostasis (CTH), University Medical \\ Center Mainz, Mainz, Germany \\ Thromb Haemost 2019;119:1547-1549.
}

Isolated distal deep vein thrombosis (IDDVT) refers to thrombi limited to the infrapopliteal deep (axial or muscular) veins of the lower limb. Although IDDVT represents a frequent finding in patients with suspected DVT, ${ }^{1}$ it has long been considered the "poor cousin" of thromboembolic events occurring at other venous sites in light of its alleged benign course. Because of the general lack of interest for this condition, there is conspicuously little evidence in the literature supporting clinical decisions (-Fig. 1). ${ }^{2}$ This in contrast to the vast literature dealing with the diagnosis and treatment of venous thromboembolism in general, essentially proximal DVT

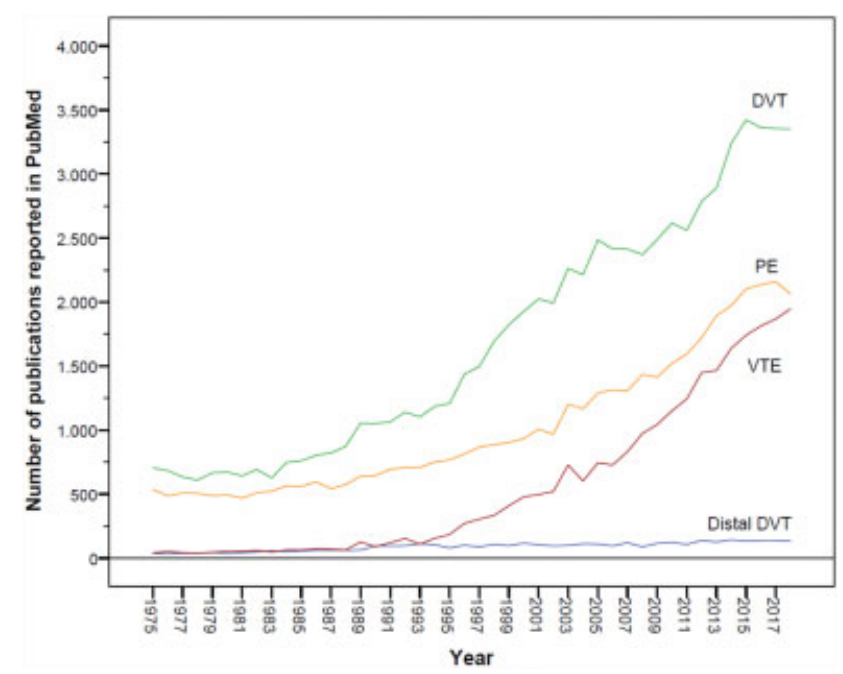

Fig. 1 Publication trends illustrating the annual number of studies reported in PubMed for different manifestations of venous thromboembolism. The literature search strategy accounted for synonyms (e.g., 'venous/vein', or'calf/distal/muscular'), popular acronyms, and different combinations of keywords. DVT, deep vein thrombosis; PE, pulmonary embolism; VTE, venous thromboembolism.

(D) Stefano Barco's ORCID is https://orcid.org/0000-0002-2618$347 X$.

received

July 23, 2019

accepted after revision

July 23, 2019

Address for correspondence Stefano Barco, MD, PhD, Center for Thrombosis and Hemostasis (CTH), University Medical Center Mainz, Langebeckstraße 1, Building 403, Room 117, 55131 Mainz, Germany (e-mail: s.barco@uni-mainz.de).

(PDVT) and pulmonary embolism (PE). ${ }^{3-9}$ It may not come as a surprise, therefore, that diagnostic and therapeutic practices for IDDVT vary across geographical regions. ${ }^{1,10,11}$

The work by Schellong et $\mathrm{al}^{12}$ provides a comprehensive description of the clinical characteristics, treatment, and course of patients diagnosed with IDDVT, PDVT, or PE enrolled in the prospective, multinational, observational Global Anticoagulant Registry in the FIELD of Venous Thromboembolism (GARFIELD-VTE; NCT02155491). ${ }^{13}$ In GARFIELD-VTE, 10,088 patients with a diagnosis of first or recurrent VTE and requiring anticoagulant treatment were included at more than 500 reference sites representative of VTE care for each of the 28 countries involved. The investigators captured data on baseline characteristics, treatment of acute VTE, hospitalizations, and clinical outcomes from the time of VTE diagnosis and during a 36-month follow-up period in the various care settings. ${ }^{13}$

One of the merits of this study is that it provides readers with an updated view of the way IDDVT is perceived and managed globally. The first striking result is the relative frequency of IDDVT diagnoses. The ratio of the number of patients with IDDVT to those with PDVT was 0.56 overall, corresponding to 56 patients being diagnosed with IDDVT every 100 PDVT diagnoses. However, extreme heterogeneity was observed across countries, with ratios ranging from 0.15 in Canada to 1.96 in Australia. ${ }^{12}$ Indeed, these figures can by no means reflect the true proportion of IDDVT, since they represent probabilities conditional to the type of screening at each center, the diagnostic strategies adopted, and the eligibility criteria of GARFIELD-VTE, for example, being treated for VTE. ${ }^{13}$ However, taking this into consideration, these results indicate that IDDVT can be frequently encountered in clinical practice and that dramatic variation exists in diagnostic patterns. This is entirely consistent with what was described more than a decade ago in an Italian survey of multiple specialized centers ${ }^{1}$ and, more recently, reported in a systematic review and meta-analysis of cohort studies on

(c) 2019 Georg Thieme Verlag KG Stuttgart · New York
DOI https://doi.org/ $10.1055 / \mathrm{s}-0039-1696983$. ISSN 0340-6245. 
isolated DVT. ${ }^{14}$ In this perspective, it appears, nothing has changed.

Indeed, the authors emphasize that this variability may be due to the intrinsic nature of their study, which was designed to contribute a snapshot of current practices and, therefore, did not include standardized diagnostic algorithms or an overt definition of IDDVT. ${ }^{12}$ These results indirectly highlight persisting uncertainty regarding (1) the anatomical level that defines "distal," (2) whether the muscle veins should be considered part of the "deep" venous system, and (3) the nomenclature used to name distal (or calf) DVT in the literature.

In this context of ambiguity, both under- and overdiagnosis of IDDVT are plausible and explain such diverse ratios of IDDVT cases. Underdiagnosis may characterize those centers adopting a strategy based on compression ultrasound scan limited to proximal veins, which would only detect subsequent proximal extensions or PE. Overdiagnosis can be expected if bilateral whole-leg compression ultrasound is routinely performed, with obvious consequences for patients with asymptomatic events, who are then exposed to unnecessary anticoagulation. ${ }^{15,16}$ An additional factor which likely influences the frequency of IDDVT diagnosis is represented by which professionals conduct diagnostic examinations. It has been shown that the accuracy achieved by trained vascular specialists, general practitioners, and nurses may vary, ${ }^{17}$ and that there are discrepancies between scanning protocols adopted by different health care professionals. ${ }^{18}$

The GARFIELD-VTE also shows that only a tiny minority of patients with VTE were assessed for their pretest clinical probability of VTE or received D-dimer measurement. ${ }^{19}$ This could be somehow expected for IDDVT, since prior studies demonstrated that diagnostic algorithms available for PDVT and PE are less accurate in patients with suspected IDDVT. ${ }^{20}$ However, in the era of extensive use of VTE imaging techniques $^{21}$ and, concurrently, of the development of diagnostic algorithms designed to rationalize their use, ${ }^{22}$ it is disappointing to observe that only $5 \%$ of the GARFIELD-VTE population underwent pretest assessment by standardized tools (e.g., the Wells' criteria). ${ }^{12}$

The present study supports prior observations suggesting that different manifestations of VTE have different etiologies. In GARFIELD-VTE, the authors confirm the potential link between IDDVT and distinct demographic characteristics or baseline risk factors, such as female sex, recent surgery or trauma, absence of cancer or prior VTE, and hormonal contraception. ${ }^{10,14,15,23}$ Furthermore, they show that the rate of newly diagnosed cancer during 1-year follow-up was lower after acute IDDVT (1.3\%) than after PDVT (2.5\%) or $\mathrm{PE}(2.6 \%)$, therefore partially contradicting findings from a prospective multicenter cohort study conducted in France. ${ }^{24}$

In GARFIELD-VTE, the distribution of concomitant risk factors for VTE and the VTE location did not appear to influence the class of the anticoagulant prescribed to patients. ${ }^{12}$ However, they had repercussions on the length of anticoagulation, which was shorter in patients with IDDVT (vs. PDVT or PE). It remains unclear, however, whether the judgment of the individual risk of recurrence was primarily driven by the higher prevalence of transient provoking risk factors or by the distal location of DVT.

This fact indirectly raises the question whether IDDVT represents an independent positive prognostic factor for the risk of recurrence and death, ${ }^{25-27}$ or if this correlation is mediated by the presence and severity of concomitant provoking risk factors for VTE. ${ }^{26,28}$ The results of GARFIELD-VTE are in line with prior studies of patients with major persistent risk factors, ${ }^{29-31}$ and show that the presence of cancer was the main determinant of outcomes. Indeed, the risk of recurrence within the first year after cancer-associated IDDVT was similar to that of patients with cancer-associated PDVT (sub-hazard ratio 1.05 adjusted for age and sex; 95\% confidence interval 0.55-2.00) and high enough ( 12-13\%) in both groups to influence the decision to extend anticoagulation beyond the first 3 months. This may not be the case for patients with IDDVT caused by transient risk factors, who were characterized by a lower risk of recurrence than PDVT (hazard ratio 0.48 adjusted for age and sex; 95\% confidence interval 0.30-0.78), amounting to a 1-year rate of 3.0\%. Patients with unprovoked IDDVT had a risk of recurrence of $5.4 \%$, similar to that of PDVT. ${ }^{12}$

We must recognize that the lack of central adjudication of clinical outcomes, the high prevalence ( $15 \%$ ) of patients with prior VTE, and the lack of adjustment for the duration of anticoagulation limit the interpretation of the results and further comparisons with prior studies focusing on first isolated DVT. While the best evidence concerning prognostic factors and efficacy of anticoagulant therapy still comes from prospective cohort studies $26,27,30,32$ and randomized controlled trials, ${ }^{33}$ the GARFIELD-VTE registry provides us with a clear take-home message. A standardized definition of IDDVT is needed, as well as a broader application of validated diagnostic algorithms and therapeutic schemes. Clear rules to better stratify patients with IDDVT based on their individual risk profile will have a major impact on the duration of anticoagulation.

\section{Funding}

This work was supported by the German Federal Ministry of Education and Research (BMBF 01E01003). The author is solely responsible for the content of this publication.

\section{Conflict of Interest}

S.B. reports personal fees from BTG, LeoPharma, and Bayer HealthCare, and nonfinancial support from Bayer HealthCare and Daiichi Sankyo, outside the submitted work. S.B. also reports personal fees from Bayer HealthCare, LeoPharma, BTG, other from Bayer HealthCare, and Daiichi Sankyo, outside the submitted work.

\section{References}

1 Palareti G, Agnelli G, Imberti D, et al; MASTER investigators. A commentary: to screen for calf DVT or not to screen? The highly variable practice among Italian centers highlights this important and still unresolved clinical option. Results from the Italian MASTER registry. Thromb Haemost 2008;99(01):241-244 
2 Kearon C, Akl EA, Ornelas J, et al. Antithrombotic therapy for VTE disease: CHEST Guideline and Expert Panel Report. Chest 2016; 149(02):315-352

3 Kalayci A, Gibson CM, Chi G, et al. Asymptomatic deep vein thrombosis is associated with an increased risk of death: insights from the APEX trial. Thromb Haemost 2018;118(12):2046-2052

4 Albertsen IE, Nielsen PB. Searching for high-risk venous thromboembolism patients using risk scores: adding to the heap or closing a gap? Thromb Haemost 2018;118(10):1686-1687

5 Roetker NS, Lutsey PL, Zakai NA, Alonso A, Adam TJ, MacLehose RF. All-cause mortality risk with direct oral anticoagulants and warfarin in the primary treatment of venous thromboembolism. Thromb Haemost 2018;118(09):1637-1645

6 Khorana AA, Weitz JI. Treatment challenges in venous thromboembolism: an appraisal of rivaroxaban studies. Thromb Haemost 2018;118(S 01):S23-S33

7 Schulman S, Ageno W, Konstantinides SV. Venous thromboembolism: past, present and future. Thromb Haemost 2017;117(07): 1219-1229

8 Cohen AT, Gitt AK, Bauersachs R, et al. The management of acute venous thromboembolism in clinical practice. Results from the European PREFER in VTE Registry. Thromb Haemost 2017;117 (07):1326-1337

9 Blondon M, Spirk D, Kucher N, et al. Comparative performance of clinical risk assessment models for hospital-acquired venous thromboembolism in medical patients. Thromb Haemost 2018; 118(01):82-89

10 Ageno W, Mantovani LG, Haas S, et al. Patient management strategies and long-term outcomes in isolated distal deep-vein thrombosis versus proximal deep-vein thrombosis: findings from XALIA. TH Open 2019;3(01):e85-e93

11 Spirk D, Husmann M, Willenberg T, et al. Inconsistencies in the planning of the duration of anticoagulation among outpatients with acute deep-vein thrombosis. Results from the OTIS-DVT Registry. Thromb Haemost 2011;105(02):239-244

12 Schellong S, Goldhaber S, Weitz J, et al. Isolated distal deep vein thrombosis: perspectives from the GARFIELD-VTE Registry. Thromb Haemost 2019;119(09):1675-1685

13 Weitz JI, Haas S, Ageno W, et al. Global Anticoagulant Registry in the Field - Venous Thromboembolism (GARFIELD-VTE). Rationale and design. Thromb Haemost 2016;116(06):1172-1179

14 Trinchero A, Scheres LJJ, Prochaska JH, et al. Sex-specific differences in the distal versus proximal presenting location of acute deep vein thrombosis. Thromb Res 2018;172:74-79

15 Galanaud JP, Sevestre-Pietri MA, Bosson JL, et al; OPTIMEV-SFMV Investigators. Comparative study on risk factors and early outcome of symptomatic distal versus proximal deep vein thrombosis: results from the OPTIMEV study. Thromb Haemost 2009;102 (03):493-500

16 Righini M, Paris S, Le Gal G, Laroche JP, Perrier A, Bounameaux H. Clinical relevance of distal deep vein thrombosis. Review of literature data. Thromb Haemost 2006;95(01):56-64

17 Mumoli N, Vitale J, Giorgi-Pierfranceschi M, et al; PRACTICUS Study Investigators. General practitioner-performed compression ultrasonography for diagnosis of deep vein thrombosis of the leg: a multicenter, prospective cohort study. Ann Fam Med 2017;15 (06):535-539
18 Shahi F, Murali K. Variations in ultrasound scanning protocols in the UK for suspected deep vein thrombosis in outpatients. Phlebology 2013;28(08):397-403

19 Ageno W, Haas S, Weitz JI, et al; GARFIELD-VTE investigators. Characteristics and management of patients with venous thromboembolism: the GARFIELD-VTE Registry. Thromb Haemost 2019;119(02):319-327

20 Sartori M, Cosmi B, Legnani C, et al. The Wells rule and D-dimer for the diagnosis of isolated distal deep vein thrombosis. JThromb Haemost 2012;10(11):2264-2269

21 Dentali F, Ageno W, Pomero F, Fenoglio L, Squizzato A, Bonzini M. Time trends and case fatality rate of in-hospital treated pulmonary embolism during 11 years of observation in Northwestern Italy. Thromb Haemost 2016;115(02):399-405

22 Righini M, Le Gal G, Bounameaux H. Venous thromboembolism diagnosis: unresolved issues. Thromb Haemost 2015;113(06): 1184-1192

23 Barco S, Klok FA, Mahé I, et al; RIETE Investigators. Impact of sex, age, and risk factors for venous thromboembolism on the initial presentation of first isolated symptomatic acute deep vein thrombosis. Thromb Res 2019;173:166-171

24 Galanaud JP, Arnoult AC, Sevestre MA, et al; OPTIMEV-SFMV Investigators. Impact of anatomical location of lower limb venous thrombus on the risk of subsequent cancer. Thromb Haemost 2014;112(06):1129-1136

25 Barco S, Corti M, Trinchero A, et al. Survival and recurrent venous thromboembolism in patients with first proximal or isolated distal deep vein thrombosis and no pulmonary embolism. JThromb Haemost 2017;15(07):1436-1442

26 Kyrle PA, Kammer M, Eischer L, et al. The long-term recurrence risk of patients with unprovoked venous thromboembolism: an observational cohort study. JThromb Haemost 2016;14(12): 2402-2409

27 Galanaud JP, Sevestre MA, Genty C, et al; OPTIMEV-SFMV investigators. Incidence and predictors of venous thromboembolism recurrence after a first isolated distal deep vein thrombosis. J Thromb Haemost 2014;12(04):436-443

28 Brateanu A, Patel K, Chagin K, et al. Probability of developing proximal deep-vein thrombosis and/or pulmonary embolism after distal deepvein thrombosis. Thromb Haemost 2016;115(03):608-614

29 Valerio L, Ambaglio C, Barone M, et al. Recurrence risk after first symptomatic distal versus proximal deep vein thrombosis according to baseline risk factors. TH Open 2019;3(01):e58-e63

30 Galanaud JP, Sevestre MA, Pernod G, et al. Long-term outcomes of cancer-related isolated distal deep vein thrombosis: the OPTIMEV study. J Thromb Haemost 2017;15(05):907-916

31 Dentali F, Pegoraro S, Barco S, et al. Clinical course of isolated distal deep vein thrombosis in patients with active cancer: a multicenter cohort study. JThromb Haemost 2017;15(09):1757-1763

32 Palareti G, Cosmi B, Lessiani G, et al. Evolution of untreated calf deep-vein thrombosis in high risk symptomatic outpatients: the blind, prospective CALTHRO study. Thromb Haemost 2010;104 (05):1063-1070

33 Righini M, Galanaud JP, Guenneguez H, et al. Anticoagulant therapy for symptomatic calf deep vein thrombosis (CACTUS): a randomised, double-blind, placebo-controlled trial. Lancet Haematol 2016; 3(12):e556-e562 